\title{
Der \\ Stannius'sche Versuch am Säugetierherzen.
}

\author{
Bemerkungen zu der von H. E. Hering \\ gegebenen Darstellung. \\ Von
}

H. Winterstein (Rostock).

In einer kürzlich erschienenen Arbeit ${ }^{1}$ ), welche den Titel führt: „Über den Stannius'sehen Versuch und seine Modifikationen am Herzen der Säugetiere und des Menschen", schreibt H. E. Hering:

S. 230: „Im Jahre 1903 veröffentlichte ich ${ }^{2}$ ) im Physiologischen Centralblatte einen modifizierten Stannius'schen Versuch am Hundeherzen .... Dieser Versuch ... ist von den verschiedensten Autoren übersehen worden, so auch von Lange nd orff, der in seinem Essay vom Jahre 1905 . . zwar in der Literaturangabe die Mitteilung anführt, aber bei seiner Besprechung der im Jahre 1904 erschienenen Abhandlung von Engelmann über den Stannius'schen Versuch meinen Versuch nicht erwähnt, wie auch nicht in seiner mit C. Lehmann im Jahre 1906 veröffentlichten Mitteilung über den Versuch von Stannius an Warmblüterherzen."

S. 232: „.. 1903 habe ich ${ }^{2}$ ) über einen Stannius'schen Versuch an mit Ringer'scher Salzlösung künstlich durchströmten Hundeherzen berichtet. „Schneidet man am schlagenden Herzen die Vorhöfe weg, so stehen die Kammern nach dem letzten Schnitt trotz fortbestehender Durchströmung einige Zeit hindurch still und schlagen nach dieser Pause seltener als vor der Vorhofabtrennung."

S. 234: „Im Jahre 1906 veröffentlichten O. Langend orff und C. Lehman ${ }^{3}$ ) die schon weiter oben erwähnte Mitteilung, betitelt: ,Der Versuch von Stannius am Warmblüterherzen." Diese Mit-

1) P flüger's Arch. Bd. 145 S. 229. 1912.

2) Physiol. Centralbl. H. 1. 11. April 1903.

3) Pflüger's Arch. Bd. 112 S. 352. 1906. 
teilung brachte über diesen Versuch im Prinzip nichts Neues; wenigstens war mir alles Wesentliche darüber schon am Säugetierherzen bekannt.

Langendorff und Lebmann haben mit dem Wegschneiden des, Venensinus' in Wirklichkeit nicht den Versuch von Stannius ausgeführt, sondern eine Modifikation desselben; es sei dies nur deswegen erwähnt, weil es aus der Mitteilung von Langendorff und Lehmann nicht klar genug hervorgeht. Das Ergebnis stimmte mit den von mir vorher veröffentlichten modifizierten Stannius'schen Versuchen am Hundeherzen überein."

Wer ohne Kenntnis des tatsächlichen Sachverhaltes diese Darstellung liest, muss etwa zu der folgenden Auffassung kommen: Im Jahre 1906 hat Langendorff Versuche veröffentlicht, die nichts wesentlich Neues brachten gegenüber solchen, die H. E. Hering bereits drei Jahre früher mitgeteilt hatte. Diese letzteren Versuche hat L a ngend orff überdies in seiner Arbeit mit keiner Silbe erwähnt. Obwohl allen, die Oskar Langendorff näher gekannt haben, seine peinliche Exaktheit, gründliche Literaturkenntnis und neidlose Anerkennung fremden Verdienstes zur Genüge bekannt sein dürfte, fühle ich mich doch verpflichtet, dieser für viele irreführenden Darstellung entgegenzutreten.

In seiner zitierten Arbeit schrieb Langendorff (S. 352): "Seit den Versuchen von v. Wittich, Wooldridge, Tigerstedt, His jun. ist ja bekannt, dass die von den Vorhöfen abgetrennten Kammern des Säugetierherzens nach einem flüchtigen Stillstand mit verlangsamtem und von dem der Atrien verschiedenem Rhythmus weiter pulsieren; welche bedeutsame Rolle bei dieser Isolierung das von His entdeckte Übergangsbündel spielt, ist besonders durch die schönen Arbeiten von H. E. Hering dargetan worden. Für uns handelte es sich aber um die Frage, ob und in welcher Weise die Tätigkeit des Herzens durch die alleinige Fortnahme des Venensinus verändert wird."

Die Beobachtung, dass die Durchschneidung zwischen Vorböfen und Kammern beim Säugetierherzen einen vorübergehenden Stillstand der letzteren zur Folge haben kann, ist also, wie ja auch aus einem von Hering selbst (S. 232) wiedergegebenen Zitat von $\mathrm{Krehl}$ und Romberg hervorgeht, schon längst vor Hering gemacht worden und selbstredend auch $\mathrm{L}$ angend orff wohl bekannt gewesen. Nun müsste man auf Grund der oben zitierten Sätze Hering's meinen, dieser habe seine im Jahre 1903 veröffentlichten Beobachtungen 
als eine "Modifikation des Stannius'schen Versuches" aufgefasst, sie etwa gar unter diesem Titel veröffentlicht. Das war keineswegs der Fall. Die Mitteilung trägt vielmehr den Titel: „Über die Wirksamkeit des Accelerans auf die von den Vorhöfen abgetrennten Kammern isolierter Säugetierherzen", und in der ganzen Mitteilung ist das Wort "Stannius'seher Versuch" nicht enthalten und auch mit keinem Worte auf irgendwelche Beziehungen zu diesem bingedeutet! Und mit Recht. Denn die Durchtrennung des Herzens an der Vorhofkammergrenze ist offenbar ein ganz anderer Eingriff als die erste Stannius'sche Ligatur und wäre höchstens mit der zweiten Ligatur vergleichbar, die ja bekanntlich gerade die entgegengesetzte Wirkung ausübt. Jetzt aber schreibt H. E. Hering (S. 230):

"Unter der Modifikation des Stannius'sehen Versuches verstehe ich dementsprechend alle Umstände, welche es bewirken, dass das Herz dem Einfluss der nomotopen Ursprungsreize entzogen wird und daraufhin unterdem Einfluss heterotoper Ursprungsreize zu schlagen anfängt."

Nun kann man über die Deutung des Stannius'schen Versuches verschiedener Meinung sein, über den Versuch selbst aber giht es keine Privatansichten. Denn der Stannius' sche Versuch ist keine Theorie, sondern ein wohlumschriebener experimenteller Eingriff; es ist eben der Versuch, den der Rostocker Physiologe Hermann Stannius zuerst im Jahre 1851 mitgeteilt hat ${ }^{1}$ ): "Wird genau diejenige Stelle unterbunden, wo der Hohlvenensinus in den rechten Vorhof mündet, sosteht das ganzeHerz im Zustande der Diastole anhaltend stille." Als eine Modifikation dieses Versuches mag man es bezeichnen, wenn man die Abtrennung des Venensinus vom Vorhof statt durch eine Ligatur durch einen Schnitt vornimmt; niemals aber kann man den Herzstillstand, der nach Durchtrennung des Herzens zwischen Vorhöfen und Kammern auftritt, d. h. nach einem Fingriff, der in den Versuchen von Stannius gerade die entgegengesetzte Wirkung hatte, das durch die erste Ligatur zum Stilistand gebrachte Herz wieder zum Schlagen zu bringen, als eine „Modifikation des Stanniusschen Versuches" bezeichnen. $O b$ beide Erscheinungen in letzter Linie auf der gleichen Ursache beruhen oder nicht, ist hierbei völlig gleichgültig.

1) Zwei Reihen physiologischer Versuche. Adler's Erben, Rostock 1851. 
158 H. Winterstein: Der Stannius'sche Versuch am Säugetierherzen.

Nun weist H. E. Hering darauf hin, dass auch Langendorff's Versuch am Warmblüterherzen nur eine "Modifikation des Stannius' schen Versuches" war, und macht ihm zum Vorwurf, dies nicht genügend deutlich hervorgehoben zu haben. Mir ist nicht recht klar, wogegen sich dieser Vorwurf richtet. Die altübliche, schon 1858 von Eckhard eingeführte Modifikation, die Abtrennung statt durch eine Ligatur, durch einen Schnitt vorzunehmen, bedurfte wohl keiner Begründung; oder sollte noch nachdrücklicher betont werden, dass es sich bloss um die Entfernung des dem Sinus entsprechenden Teiles der Vorhofswand handelte? Eine solche Hervorhebung wäre wohl nur für jene notwendig gewesen, welche mit den Anfangsgründen der Anatomie des Säugetierherzens nicht vertraut sind; denn alle die anderen, welche wissen, dass das Säugetierherz einen gesonderten Venensinus überhaupt nicht besitzt, brauchte man wohl nicht erst darauf aufmerksam zu machen, dass der Versuch hier nicht in der Entfernung eines gar nicht existierenden Herzabschnittes, sondern bloss desjenigen Herzteiles bestehen könne, der vergleichend-anatomisch und entwicklungsgeschichtlich dem Venensinus des Froschherzens entspricht.

Die Entdeckung von Langend orff und Lehmann, dass diese Stelle sich nicht bloss dadurch wahrscheinlich "als Ursprungsort der normalen Herzreize und Ausgangspunkt der Herzbewegung beim Säugetierherzen" dokumentiert, dass, wie die von Langendorff und Adam ausgeführten Versuche dargetan hatten, von hier aus der Rhythmus der ganzen Herztätigkeit beeinflusst werden kann, sondern auch dadurch, dass seine Entfernung einen vorübergehenden Herzstillstand herbeizuführen vermag, hat also in Wahrheit eine ganz neue und bedeutungsolle Tatsache zutage gefördert.

H. E. Hering kommt in seiner Arbeit zu der bemerkenswerten Schlussfolgerung (S. 248): „Der Stannius'sche Versuch (auch erste Stannius'sche Ligatur genannt) ist nur ein Spezialfall des Verhaltens des Herzens, wenn es dem Einflusse der nomotopen Ursprungsreize entzogen wird." Um so sonderbarer ist die vollständige Umkehrung des Sachverhaltes, die Hering vornimmt, wenn er von dieser Schlussfolgerung aus rückwärtsgehend die Bedeutung und Originalität jener Versuche zu schmälern sucht, auf die sich diese Schlussfolgerung in letzter Linie auf baut. 